\title{
Analytical approach for fractional extended Fisher-Kolmogorov equation with Mittag-Leffler kernel
}

\author{
P. Veeresha', D.G. Prakasha², Jagdev Singh³ ${ }^{2}$ llyas Khan ${ }^{4 *}$ and Devendra Kumar ${ }^{5}$
}

\section{"Correspondence:}

ilyaskhan@tdtu.edu.vn

${ }^{4}$ Faculty of Mathematics and Statistics, Ton DucThang University, Ho Chi Minh City, Vietnam Full list of author information is available at the end of the article

\section{Springer}

\begin{abstract}
A new solution for fractional extended Fisher-Kolmogorov (FEFK) equation using the $q$-homotopy analysis transform method ( $q$-HATM) is obtained. The fractional derivative considered in the present work is developed with Atangana-Baleanu (AB) operator, and the technique we consider is a mixture of the $q$-homotopy analysis scheme and the Laplace transform. The fixed point hypothesis is considered for the existence and uniqueness of the obtained solution of this model. For the validation and effectiveness of the projected scheme, we analyse the FEFK equation in terms of arbitrary order for the two distinct cases. Moreover, numerical simulation is demonstrated, and the nature of the achieved solution in terms of plots for distinct arbitrary order is captured.
\end{abstract}

Keywords: Extended Fisher-Kolmogorov equation; Atangana-Baleanu derivative; Fixed point theorem; Laplace transform; $q$-Homotopy analysis method

\section{Introduction}

The concept of fractional calculus (FC) is as old as the classical calculus. Even though its roots are planted in the period of Newton, recently it has magnetized the attention of a class of mathematicians and scientists. More precisely, the intriguing leaps of evolution and innovation in the associated fields of science and technology are found from the last thirty years within the frame of FC. There have been diverse definitions for the differential and integral with arbitrary order suggested by many pioneers in order to overcome the limitation of the previous definition, and this orientation lays the foundation [1-6]. Fractional calculus is comprehensively applied to investigate the nature and corresponding consequences of various phenomena, for instance, chaos theory [7], human diseases [8], optics [9], nanotechnology [10], and other areas [11-14].

The main purpose of studying the concept of FC is the heterogeneities phenomenon associated with complexities. Also it is proved that FC is the most efficient weapon to illustrate the mechanism related to the diffusion process since the integer order calculus is unable to capture the interesting behaviour of complex and nonlinear model related to time, history and their corresponding consequence. However, recently many researchers have proved and illustrated that the fractional calculus is able to describe these essential

(c) The Author(s) 2020. This article is licensed under a Creative Commons Attribution 4.0 International License, which permits use, sharing, adaptation, distribution and reproduction in any medium or format, as long as you give appropriate credit to the original author(s) and the source, provide a link to the Creative Commons licence, and indicate if changes were made. The images or other third party material in this article are included in the article's Creative Commons licence, unless indicated otherwise in a credit line to the material. If material is not included in the article's Creative Commons licence and your intended use is not permitted by statutory regulation or exceeds the permitted use, you will need to obtain permission directly from the copyright holder. To view a copy of this licence, visit http://creativecommons.org/licenses/by/4.0/. 
properties. Moreover, the authors [15-30] considered newly defined fractional operators in order to analyse and capture the simulating nature of various phenomena. For instance, the authors in [23] considered the fractional operator derived with the aid of Mittag-Leffler function in order to analyse the outbreak of dengue fever and presented some interesting results. The optimal control of tuberculosis and diabetes co-existence was investigated in [24], and the model of spring pendulum was analysed in [27] with the help of fractional calculus for different kernels. Many young researchers began to study generalised calculus due to rapid growth in the computer software with mathematical algorithms in order to examine the diverse class of complex phenomena and execute their viewpoints.

We investigate an EFK equation in the present study, and the EFK equation was suggested by Coullet, Elphick and Repauxin in 1987 [31], and later, in 1988, Dee and Saarloos $[32,33]$ proposed the generalization of the standard FK equation. Here, we consider the EFK equation $[34,35]$

$$
u_{t}+\mu \Delta^{2} u-\Delta u+\phi(u)=0, \quad x \in \Omega, t \in(0, T]
$$

where $\phi(u)=u^{3}-u, T>0, \Omega \in(0,1)$ with boundary $\partial \Omega$ and $\mu$ signifies a positive constant. For $\mu=0$, Eq. (1) reduces to the classical FK equation. Including fourth-order term to the classical FK equation, the authors in [31] illustrated and natured Eq. (1). This term plays an important role in phase transitions near critical points (Lipschitz points).

The considered model has diverse significance, it has been analysed and also illustrated by many researchers associated with science and technology. For instance, near to Lipschitz point the mesoscopic model of a phase transition was demonstrated by the authors in [36], the considered model describes the reaction-diffusion system by travelling waves [37]; in liquid crystals the authors in [38] illustrated the propagation of domain walls, pattern formation in bi-stable systems [32].

Many interesting and nonlinear models arising in associated fields of science and engineering have been effectively and systematically exemplified with the aid of generalised calculus in the present scenario. Many elder researchers suggested the distinct definition for both integral and differential operators having fractional order. Nevertheless, each basic notion has its own confines. The importance of the initial conditions is not described by Riemann-Liouville derivative, the singular kernel is not associated with the notion of fractional calculus described by Caputo. In order to overcome the above-mentioned limitations, Caputo and Fabrizio in 2015 defined the operator [39], and later many authors employed it to investigate and present some interesting behaviour for nonlinear complex problems. Recently, many researchers pointed out some issues related to essential properties describing the behaviour of nonlinear problems like the non-local and non-singular kernel. In order to overcome these limitations with the help of Mittag-Leffler functions, Atangana and Baleanu derived the new fractional derivative in 2016, namely AtanganaBaleanu (AB) derivative [40]. This derivative buried all the above-mentioned issues.

On the other hand, it is essential and very important to evaluate the solution for the integral and differential equations describing the above mechanisms. In connection with this, physicist and mathematicians established more accurate and very effective techniques. There are numerous methods available in the literature, for instance, decomposition method, perturbation methods, homotopy methods, iteration methods and many others. Among these schemes, the homotopy analysis method (HAM) [41, 42] has been 
extensively considered by many researchers due to its applicability, efficiency and accuracy. HAM has been employed to nonlinear problems for the purpose of examining the behaviour without linearization, transformation, discretization or perturbation. However, it necessitates huge computer memory and time, and hence the combination of HAM with well-established transform algorithm is imposed.

Here, we consider FEFK equation of the form

$$
{ }_{a}^{A B C} D_{t}^{\alpha} u(x, t)+\mu \Delta^{2} u-\Delta u+\phi(u)=0, \quad 0<\alpha \leq 1,
$$

where $\alpha$ is fractional order. In this paper, we consider the improved method of HAM with the elegant amalgamation of Laplace transform in order to reduce huge computation and computer memory [43]. Due to efficacy and reliability, $q$-HATM has been applied to many nonlinear problems as well as models which describe the various phenomena by many researchers in order to present the nature, capture the behaviour and to illustrate the corresponding consequences [44-51]. The novelty of the considered algorithm is that it is fabricated with auxiliary and homotopy parameters, and these can help quick convergence in the obtained solution. Also, it provides a simple computational scheme to find the analytical solution. Further, it offers more freedom to consider the equation type nonlinear problems and a distinct class of initial conditions. The proposed solution procedure can preserve more exactness while reducing the huge computational work and time in comparison with other traditional schemes.

The projected model has fascinated the consideration of many authors since it plays a substantial role in describing various nonlinear models. Recently, many researchers have found and analysed the solution with the help of distinct methods. For instance, the authors in [52] found the heteroclinic solutions for Eq. (1) by variational algorithm; the authors in [53] employed the finite difference method and also presented existence and stability for the corresponding solution; the attractor bifurcation was illustrated by the authors in [54] for the proposed model; the authors in [55] presented the global dynamics of stationary solutions for Eq. (1); the periodic solution was obtained by the authors in [56]; the Fourier pseudo-spectral scheme was employed by the researchers in [35] to presented some simulating consequences of the results to understand the nature of the considered nonlinear problem.

In this paper, the equation describing the phase transitions near critical points, called EFK equation, is considered. In order to integrate the memory effect and essential properties like kernel and non-singularity, we generalise the considered nonlinear model by replacing time derivative with fractional derivative with the aid of $\mathrm{AB}$ operator. Moreover, with the assistance of fixed point hypothesis, the existence and uniqueness are presented for the solution of the considered problem. Moreover, we consider two different cases in order to demonstrate the applicability and the efficiency of the considered method. We present the numerical simulation in order to illustrate the accuracy of $q$-HATM. The remaining part of the paper is organised as follows: the essential fundamentals and basic notions are defined in Sect. 2; the solution procedure of the projected scheme is presented in Sect. 3, and in Sect. 4 we present the solution for the considered problem with the aid of $q$ HATM. The existence and uniqueness for the solution of FEFK equation are presented in Sect. 5 with the help of fixed point theorem. Further, the numerical results and discussion for two different cases and conclusion on the obtained results are respectively presented in Sect. 6 and Sect. 7. 


\section{Preliminaries}

Here, we present the fundamental notions of fractional derivative and Laplace transform [57-61].

Definition 1 The Atangana-Baleanu-Caputo (ABC) derivative for the function $f \in$ $H^{1}(a, b)(b>a, \alpha \in[0,1])$ is presented as follows [40]:

$$
{ }_{a}^{A B C} D_{t}^{\alpha}(f(t))=\frac{\mathcal{B}[\alpha]}{1-\alpha} \int_{a}^{t} f^{\prime}(\vartheta) E_{\alpha}\left[-\alpha \frac{(t-\vartheta)^{\alpha}}{1-\alpha}\right] d \vartheta .
$$

Definition 2 The fractional-order AB integral of a function $f \in H^{1}(a, b), b>a, \alpha \in[0,1]$, and then its fractional-order in Riemann-Liouville (RL) sense is defined as follows [40]:

$$
{ }_{a}^{A B R} D_{t}^{\alpha}(f(t))=\frac{\mathcal{B}[\alpha]}{1-\alpha} \frac{d}{d t} \int_{a}^{t} f(\vartheta) E_{\alpha}\left[\alpha \frac{(t-\vartheta)^{\alpha}}{\alpha-1}\right] d \vartheta
$$

Definition 3 The AB integral of fractional order is presented as follows [40]:

$$
{ }_{a}^{A B} I_{t}^{\alpha}(f(t))=\frac{1-\alpha}{\mathcal{B}[\alpha]} f(t)+\frac{\alpha}{\mathcal{B}[\alpha] \Gamma(\alpha)} \int_{a}^{t} f(\vartheta)(t-\vartheta)^{\alpha-1} d \vartheta .
$$

Definition 4 As reference to $\mathrm{AB}$ derivative of the function $f(t)$, the Laplace transform (LT) of $f(t)$ is presented as follows [40]:

$$
L\left[{ }_{0}^{A B C} D_{t}^{\alpha}(f(t))\right](s)=\frac{\mathcal{B}[\alpha]}{1-\alpha} \frac{s^{\alpha} L[f(t)]-s^{\alpha-1} f(0)}{s^{\alpha}+(\alpha /(1-\alpha))} .
$$

Theorem 1 The Lipschitz condition holds for the ABR and ABC derivatives [40]

$$
\left\|_{a}^{A B R} D_{t}^{\alpha} f_{1}(t)-{ }_{a}^{A B R} D_{t}^{\alpha} f_{2}(t)\right\|<K_{2}\left\|f_{1}(x)-f_{2}(x)\right\|
$$

and

$$
\left\|{ }_{a}^{A B C} D_{t}^{\alpha} f_{1}(t)-{ }_{a}^{A B C} D_{t}^{\alpha} f_{2}(t)\right\|<K_{1}\left\|f_{1}(x)-f_{2}(x)\right\| .
$$

Theorem 2 The fractional differential equation ${ }_{a}^{A B C} D_{t}^{\alpha} f_{1}(t)=s(t)$ has a unique solution, which is presented as follows [40]:

$$
f(t)=\frac{(1-\alpha)}{\mathcal{B}[\alpha]} s(t)+\frac{\alpha}{\mathcal{B}[\alpha] \Gamma(\alpha)} \int_{0}^{t} s(\varsigma)(t-\varsigma)^{\alpha-1} d \varsigma .
$$

\section{Fundamental procedure of projected scheme}

In this part, we take a fractional differential equation to present the solution procedure of the projected method [62-64]

$$
{ }_{a}^{A B C} D_{t}^{\alpha} v(x, t)+\mathcal{R} v(x, t)+\mathcal{N} v(x, t)=f(x, t), \quad n-1<\alpha \leq n,
$$

with the initial condition

$$
v(x, 0)=\mathfrak{g}(x)
$$


where ${ }_{a}^{A B C} D_{t}^{\alpha} v(x, t)$ symbolises the $\mathrm{AB}$ derivative of $v(x, t)$. By applying $L T$ on Eq. (10), one can get

$$
\mathcal{L}[v(x, t)]-\frac{\mathfrak{g}(x)}{s}+\frac{1}{\mathcal{B}[\alpha]}\left(1-\alpha+\frac{\alpha}{s^{\alpha}}\right)\{\mathcal{L}[\mathcal{N} v(x, t)]+\mathcal{L}[\mathcal{R} v(x, t)]-\mathcal{L}[f(x, t)]\}=0 .
$$

Now, corresponding to foregoing equations, the nonlinear operator is presented as follows:

$$
\begin{aligned}
\mathcal{N} & {[\varphi(x, t ; q)] } \\
= & \mathcal{L}[\varphi(x, t ; q)]-\frac{\mathfrak{g}(x)}{s} \\
& +\frac{1}{\mathcal{B}[\alpha]}\left(1-\alpha+\frac{\alpha}{s^{\alpha}}\right)\{\mathcal{L}[\mathcal{R} \varphi(x, t ; q)]+L[\mathcal{N} \varphi(x, t ; q)]-L[f(x, t)]\},
\end{aligned}
$$

where $q \in\left[0, \frac{1}{\mathfrak{n}}\right]$. Now, the homotopy is presented as follows with non-zero auxiliary parameter $\hbar$ and embedding parameter $q \in\left[0, \frac{1}{n}\right](n \geq 1)$ :

$$
\hbar q \mathcal{N}[\varphi(x, t ; q)]=(1-\mathfrak{n} q) \mathcal{L}\left[\varphi(x, t ; q)-v_{0}(x, t)\right]
$$

where $L$ signifies $L T$. For $q=0$ and $q=\frac{1}{n}$, the following are satisfied:

$$
\varphi(x, t ; 0)=v_{0}(x, t), \quad \varphi\left(x, t ; \frac{1}{n}\right)=v(x, t)
$$

By increasing $q$ from 0 to $\frac{1}{\mathfrak{n}}$, then $\varphi(x, t ; q)$ converges from $v_{0}(x, t)$ to $v(x, t)$. Then, with the help of Taylor's theorem near to $q$, one can have

$$
\varphi(x, t ; q)=v_{0}(x, t)+\sum_{m=1}^{\infty} v_{m}(x, t) q^{m},
$$

where

$$
v_{m}(x, t)=\left.\frac{1}{m !} \frac{\partial^{m} \varphi(x, t ; q)}{\partial q^{m}}\right|_{q=0} .
$$

For the proper choice of $v_{0}(x, t), n$ and $\hbar$, the series (16) converges at $q=\frac{1}{n}$. Then

$$
v(x, t)=v_{0}(x, t)+\sum_{m=1}^{\infty} v_{m}(x, t)\left(\frac{1}{n}\right)^{m} .
$$

Multiplying by $\frac{1}{m !}$ after differentiating Eq. (14) $m$-times with $q$ and then putting $q=0$, one gets

$$
\mathcal{L}\left[v_{m}(x, t)-\mathrm{k}_{m} v_{m-1}(x, t)\right]=\hbar R_{m}\left(\vec{v}_{m-1}\right)
$$

and later we define vectors as

$$
\vec{v}_{m}=\left\{v_{0}(x, t), v_{1}(x, t), \ldots, v_{m}(x, t)\right\} .
$$


On applying inverse $L T$ to Eq. (19), we obtain

$$
v_{m}(x, t)=\hbar \mathcal{L}^{-1}\left[R_{m}\left(\vec{v}_{m-1}\right)\right]+\mathrm{k}_{m} v_{m-1}(x, t),
$$

where

$$
\begin{aligned}
R_{m}\left(\vec{v}_{m-1}\right)= & L\left[v_{m-1}(x, t)\right]-\left(1-\frac{\mathrm{k}_{m}}{n}\right)\left(\frac{\mathfrak{g}(x)}{s}+\frac{1}{\mathcal{B}[\alpha]}\left(1-\alpha+\frac{\alpha}{s^{\alpha}}\right) L[f(x, t)]\right) \\
& +\frac{1}{\mathcal{B}[\alpha]}\left(1-\alpha+\frac{\alpha}{s^{\alpha}}\right) L\left[R v_{m-1}+\mathcal{H}_{m-1}\right]
\end{aligned}
$$

and

$$
\mathrm{k}_{m}= \begin{cases}0, & m \leq 1 \\ n, & m>1\end{cases}
$$

In Eq. (22), $\mathcal{H}_{m}$ is a homotopy polynomial, which is defined as

$$
\mathcal{H}_{m}=\frac{1}{m !}\left[\frac{\partial^{m} \varphi(x, t ; q)}{\partial q^{m}}\right]_{q=0} \quad \text { and } \quad \varphi(x, t ; q)=\varphi_{0}+q \varphi_{1}+q^{2} \varphi_{2}+\cdots
$$

With the assistance of Eqs. (23) and (24), we have

$$
\begin{aligned}
v_{m}(x, t)= & \left(\mathrm{k}_{m}+\hbar\right) v_{m-1}(x, t)-\left(1-\frac{\mathrm{k}_{m}}{n}\right) \mathcal{L}^{-1}\left(\frac{\mathfrak{g}(x)}{s}+\frac{1}{\mathcal{B}[\alpha]}\left(1-\alpha+\frac{\alpha}{s^{\alpha}}\right) L[f(x, t)]\right) \\
& +\hbar \mathcal{L}^{-1}\left\{\frac{1}{\mathcal{B}[\alpha]}\left(1-\alpha+\frac{\alpha}{s^{\alpha}}\right) L\left[R v_{m-1}+\mathcal{H}_{m-1}\right]\right\} .
\end{aligned}
$$

Then we can find the terms of $v_{m}(x, t)$ with the aid of Eq. (25). The $q$-HATM solution is written as follows:

$$
v(x, t)=\sum_{m=0}^{\infty} v_{m}(x, t)
$$

\section{Solution for FEFK equation}

Here, we examine the FEFK equation presented in Eq. (2) to find its solution with the assistance of the projected scheme

$$
{ }_{a}^{A B C} D_{t}^{\alpha} u(x, y, t)+\mu \Delta^{2} u-\Delta u+u^{3}-u=0, \quad 0<\alpha \leq 1,
$$

with the initial conditions (ICs)

$$
u(x, y, 0)=u_{0}(x, y, t)
$$

Taking $L T$ on Eq. (27) and with the aid of Eq. (28), one can have

$$
L[u(x, y, t)]=\frac{1}{s}\left(u_{0}(x, y, t)\right)+\frac{1}{\mathcal{B}[\alpha]}\left(1-\alpha+\frac{\alpha}{s^{\alpha}}\right) L\left\{\mu \Delta^{2} u-\Delta u+u^{3}-u\right\} .
$$


Now, we present the nonlinear operator $N$ as follows:

$$
\begin{aligned}
N[\varphi(x, y, t ; q)]= & L[\varphi(x, y, t ; q)]-\frac{1}{s}\left(u_{0}(x, y, t)\right) \\
& +\frac{1}{\mathcal{B}[\alpha]}\left(1-\alpha+\frac{\alpha}{s^{\alpha}}\right) \\
& \times L\left\{\mu \Delta^{2} \varphi(x, y, t ; q)-\Delta \varphi(x, y, t ; q)+\varphi(x, y, t ; q)^{3}-\varphi(x, y, t ; q)\right\} .
\end{aligned}
$$

At $\mathcal{H}(x, y, t)=1$, the $m$ th order deformation equation by using $q$-HATM is expressed as

$$
L\left[u_{m}(x, y, t)-\mathrm{k}_{m} u_{m-1}(x, y, t)\right]=\hbar R_{m}\left[\vec{u}_{m-1}\right]
$$

where

$$
\begin{aligned}
R_{m}\left[\vec{u}_{m-1}\right]= & L\left[u_{m-1}(x, y, t)\right]-\left(1-\frac{\mathrm{k}_{m}}{\mathfrak{n}}\right)\left\{\frac{1}{s}\left(u_{0}(x, y, t)\right)\right\} \\
& +\frac{1}{\mathcal{B}[\alpha]}\left(1-\alpha+\frac{\alpha}{s^{\alpha}}\right) \\
& \times L\left\{\mu \Delta^{2} u_{m-1}-\Delta^{2} u_{m-1}+\sum_{j=0}^{i} \sum_{i=0}^{m-1} u_{j} u_{i-j} u_{m-1-i}-u_{m-1}\right\} .
\end{aligned}
$$

By utilizing the inversion of $L T$ on Eq. (31), we get

$$
u_{m}(x, y, t)=\mathrm{k}_{m} u_{m-1}(x, y, t)+\hbar L^{-1}\left\{R_{m}\left[\vec{u}_{m-1}\right]\right\} .
$$

On solving the preceding equations by using $u_{0}(x, y, t)$, we can obtain the terms of

$$
u(x, y, t)=u_{0}(x, y, t)+\sum_{m=1}^{\infty} u_{m}(x, y, t)\left(\frac{1}{n}\right)^{m} .
$$

\section{Existence and uniqueness of solution}

Here, with the aid of fixed point theory, we demonstrate the existence and uniqueness for the solution of the considered problem. Now, by the aid of Eq. (27), one can have

$$
{ }_{0}^{A B C} D_{t}^{\alpha}[u(x, y, t)]=\mathcal{G}(x, y, t, u) .
$$

With the help of Eq. (35) and Theorem 2, one can get

$$
u(x, y, t)-u(x, y, 0)=\frac{(1-\alpha)}{\mathcal{B}(\alpha)} \mathcal{G}(x, y, t, u)+\frac{\alpha}{\mathcal{B}(\alpha) \Gamma(\alpha)} \int_{0}^{t} \mathcal{G}(x, y, \zeta, u)(t-\zeta)^{\alpha-1} d \zeta
$$

Theorem 3 The kernel $\mathcal{G}$ satisfies the Lipschitz condition and also contraction if it satisfies $0 \leq\left(\mu \Delta^{2}-\Delta+\left(a^{2}+b^{2}+a b\right)-1\right)<1$. 
Proof Let us choose two functions $u$ and $u_{1}$ to verify the required condition, therefore we get

$$
\begin{aligned}
\left\|\mathcal{G}(x, y, t, u)-\mathcal{G}\left(x, y, t, u_{1}\right)\right\|= & \| \mu \Delta^{2}\left[u(x, y, t)-u\left(x, y, t_{1}\right)\right]-\Delta\left[u(x, y, t)-u\left(x, y, t_{1}\right)\right] \\
& +\left[u^{3}(x, y, t)-u^{3}\left(x, y, t_{1}\right)\right]-\left[u(x, y, t)-u\left(x, y, t_{1}\right)\right] \| \\
\leq & \left\|\mu \Delta^{2}-\Delta+\left(a^{2}+b^{2}+a b\right)-1\right\|\left\|u(x, y, t)-u\left(x, y, t_{1}\right)\right\| \\
\leq & \left(\mu \Delta^{2}-\Delta+\left(a^{2}+b^{2}+a b\right)-1\right)\left\|u(x, y, t)-u\left(x, y, t_{1}\right)\right\| .
\end{aligned}
$$

Since $u$ and $u_{1}$ are bounded and here $a=\|u\|$ and $b=\left\|u_{1}\right\|$. Inserting $\eta=\mu \Delta^{2}-\Delta+\left(a^{2}+\right.$ $\left.b^{2}+a b\right)-1$ in Eq. (37), we get

$$
\left\|\mathcal{G}(x, y, t, u)-\mathcal{G}\left(x, y, t, u_{1}\right)\right\| \leq \eta\left\|u(x, y, t)-u\left(x, y, t_{1}\right)\right\|
$$

It is clear that the Lipschitz condition is achieved for $\mathcal{G}_{1}$. Moreover, if $0 \leq\left(\mu \Delta^{2}-\Delta+\left(a^{2}+\right.\right.$ $\left.\left.b^{2}+a b\right)-1\right)<1$, then it leads to contraction. Now, the recursive form is present for the above relation and initial condition as follows:

$$
u_{n}(x, y, t)=\frac{(1-\alpha)}{\mathcal{B}(\alpha)} \mathcal{G}\left(x, y, t, u_{n-1}\right)+\frac{\alpha}{\mathcal{B}(\alpha) \Gamma(\alpha)} \int_{0}^{t} \mathcal{G}\left(x, y, \zeta, u_{n-1}\right)(t-\zeta)^{\alpha-1} d \zeta
$$

and

$$
u(x, y, 0)=u_{0}(x, y, t) .
$$

The difference between successive terms is defined as follows:

$$
\begin{aligned}
\phi_{n}(x, y, t)= & u_{n}(x, y, t)-u_{n-1}(x, y, t) \\
= & \frac{(1-\alpha)}{\mathcal{B}(\alpha)}\left(\mathcal{G}_{1}\left(x, y, t, u_{n-1}\right)-\mathcal{G}\left(x, y, t, u_{n-2}\right)\right) \\
& +\frac{\alpha}{\mathcal{B}(\alpha) \Gamma(\alpha)} \int_{0}^{t} \mathcal{G}\left(x, y, \zeta, u_{n-1}\right)(t-\zeta)^{\alpha-1} d \zeta .
\end{aligned}
$$

Notice that

$$
u_{n}(x, y, t)=\sum_{i=1}^{n} \phi_{i}(x, y, t)
$$

By employing the norm on Eq. (41) and considering Eq. (38), we have

$$
\left\|\phi_{n}(x, y, t)\right\| \leq \frac{(1-\alpha)}{\mathcal{B}(\alpha)} \eta\left\|\phi_{(n-1)}(x, y, t)\right\|+\frac{\alpha}{\mathcal{B}(\alpha) \Gamma(\alpha)} \eta \int_{0}^{t}\left\|\phi_{(n-1)}(x, y, \zeta)\right\| d \zeta .
$$

With the aid of foregoing results, we prove the following theorems. 
Theorem 4 For the projected system (27), the solution will exist and be unique for a particular $t_{0}$ such that

$$
\frac{(1-\alpha)}{\mathcal{B}(\alpha)} \eta+\frac{\alpha}{\mathcal{B}(\alpha) \Gamma(\alpha)} \eta<1
$$

Proof Let $u(x, y, t)$ be a bounded function sustaining the Lipschitz condition. With the assistance of Eq. (43), we get

$$
\left\|\phi_{i}(x, y, t)\right\| \leq\left\|u_{n}(x, y, 0)\right\|\left[\frac{(1-\alpha)}{\mathcal{B}(\alpha)} \eta+\frac{\alpha}{\mathcal{B}(\alpha) \Gamma(\alpha)} \eta\right]^{n} .
$$

This proves the continuity and existence of the achieved solution. Further, to verify that the above equation is the solution for Eq. (27), we consider

$$
u(x, y, t)-u(x, y, 0)=u_{n}(x, y, t)-\mathcal{K}_{n}(x, y, t) .
$$

Then, we consider achieving the required result

$$
\begin{aligned}
\left\|\mathcal{K}_{n}(x, y, t)\right\|= & \| \frac{(1-\alpha)}{\mathcal{B}(\alpha)}\left(\mathcal{G}(x, y, t, u)-\mathcal{G}\left(x, y, t, u_{n-1}\right)\right) \\
& +\frac{\alpha}{\mathcal{B}(\alpha) \Gamma(\alpha)} \int_{0}^{t}(t-\zeta)^{\mu-1}\left(\mathcal{G}(x, y, \zeta, u)-\mathcal{G}\left(x, y, \zeta, u_{n-1}\right)\right) d \zeta \| \\
\leq & \frac{(1-\alpha)}{\mathcal{B}(\alpha)}\left\|\mathcal{G}(x, y, \zeta, u)-\mathcal{G}\left(x, y, \zeta, u_{n-1}\right)\right\| \\
& +\frac{\alpha}{\mathcal{B}(\alpha) \Gamma(\alpha)} \int_{0}^{t}\left\|\mathcal{G}(x, y, \zeta, u)-\mathcal{G}\left(x, y, \zeta, u_{n-1}\right)\right\| d \zeta \\
\leq & \frac{(1-\alpha)}{\mathcal{B}(\alpha)} \eta_{1}\left\|u-u_{n-1}\right\|+\frac{\alpha}{\mathcal{B}(\alpha) \Gamma(\alpha)} \eta_{1}\left\|u-u_{n-1}\right\| t .
\end{aligned}
$$

Similarly, at $t_{0}$ we can obtain

$$
\left\|\mathcal{K}_{n}(x, y, t)\right\| \leq\left(\frac{(1-\alpha)}{\mathcal{B}(\alpha)}+\frac{\alpha t_{0}}{\mathcal{B}(\alpha) \Gamma(\alpha)}\right)^{n+1} \eta^{n+1} M
$$

We have from Eq. (47), for $n$ tending to $\infty,\left\|\mathcal{K}_{n}(x, y, t)\right\|$ approaches 0 . Now, it is important to present the uniqueness for the obtained solution. Suppose that $u^{*}(t)$ is a different solution, then one can get

$$
\begin{aligned}
u(x, y, t)-u^{*}(x, y, t)= & \frac{(1-\alpha)}{\mathcal{B}(\alpha)}\left(\mathcal{G}(x, y, t, u)-\mathcal{G}\left(x, y, t, u^{*}\right)\right) \\
& +\frac{\alpha}{\mathcal{B}(\alpha) \Gamma(\alpha)} \int_{0}^{t}\left(\mathcal{G}(x, y, \zeta, u)-\mathcal{G}\left(x, y, \zeta, u^{*}\right)\right) d \zeta .
\end{aligned}
$$


With the help of properties of the norm, Eq. (48) reduces to

$$
\begin{aligned}
\left\|u(x, y, t)-u^{*}(x, y, t)\right\|= & \| \frac{(1-\alpha)}{\mathcal{B}(\alpha)}\left(\mathcal{G}(x, y, t, u)-\mathcal{G}\left(x, y, t, u^{*}\right)\right) \\
& +\frac{\alpha}{\mathcal{B}(\alpha) \Gamma(\alpha)} \int_{0}^{t}\left(\mathcal{G}(x, y, \zeta, u)-\mathcal{G}\left(x, y, \zeta, u^{*}\right)\right) d \zeta \| \\
\leq & \frac{(1-\alpha)}{\mathcal{B}(\alpha)} \eta\left\|u(x, y, t)-u^{*}(x, y, t)\right\| \\
& +\frac{\alpha}{\mathcal{B}(\alpha) \Gamma(\alpha)} \eta t\left\|u(x, y, t)-u^{*}(x, y, t)\right\| .
\end{aligned}
$$

On simplification

$$
\left\|u(x, y, t)-u^{*}(x, y, t)\right\|\left(1-\frac{(1-\alpha)}{\mathcal{B}(\alpha)} \eta-\frac{\alpha}{\mathcal{B}(\alpha) \Gamma(\alpha)} \eta t\right) \leq 0 .
$$

From the above condition, it is clear that $u(t)=u^{*}(t)$, if

$$
\left(1-\frac{(1-\alpha)}{\mathcal{B}(\alpha)} \eta-\frac{\alpha}{\mathcal{B}(\alpha) \Gamma(\alpha)} \eta t\right) \geq 0 .
$$

Hence, Eq. (51) shows our required result.

Theorem 5 Let $(B[0, T],\|\cdot\|)$ be a Banach space. Suppose that $u_{n}(x, y, t)$ and $u(x, y, t)$ define $B$, if $0<\lambda<1$, then the series solution presented in Eq. (26) tends towards the solution of $E q$. (10).

Proof Let $\left\{\mathcal{S}_{n}\right\}$ be a partial sum of Eq. (26), then we need to show that $\left\{\mathcal{S}_{n}\right\}$ is a Cauchy sequence in $(B[0, T],\|\cdot\|)$. Then

$$
\begin{aligned}
\left\|\mathcal{S}_{n+1}(x, y, t)-\mathcal{S}_{n}(x, y, t)\right\| & =\left\|u_{n+1}(x, y, t)\right\| \\
& \leq \lambda\left\|u_{n}(x, y, t)\right\| \\
& \leq \lambda^{2}\left\|u_{n-1}(x, y, t)\right\| \leq \cdots \leq \lambda^{n+1}\left\|u_{0}(x, y, t)\right\| .
\end{aligned}
$$

For every $n, m \in N(m \leq n)$, we have

$$
\begin{aligned}
\left\|\mathcal{S}_{n}-\mathcal{S}_{m}\right\| & =\left\|\left(\mathcal{S}_{m+1}-\mathcal{S}_{m}\right)+\cdots+\left(\mathcal{S}_{n-1}-\mathcal{S}_{n-2}\right)+\left(\mathcal{S}_{n}-\mathcal{S}_{n-1}\right)\right\| \\
& \leq\left\|\mathcal{S}_{m+1}-\mathcal{S}_{m}\right\|+\cdots+\left\|\mathcal{S}_{n-1}-\mathcal{S}_{n-2}\right\|+\left\|\mathcal{S}_{n}-\mathcal{S}_{n-1}\right\| \\
& \leq\left(\lambda^{n}+\lambda^{n-1}+\cdots+\lambda^{m+1}\right)\left\|u_{0}\right\| \\
& \leq \lambda^{m+1}\left(1+\lambda+\cdots++\lambda^{n-m-2}+\lambda^{n-m-1}\right)\left\|u_{0}\right\| \\
& \leq\left(\frac{1-\lambda^{n-m}}{1-\lambda}\right) \lambda^{m+1}\left\|u_{0}\right\| .
\end{aligned}
$$

But $0<\lambda<1$, therefore $\lim _{n, m \rightarrow \infty}\left\|\mathcal{S}_{n}-\mathcal{S}_{m}\right\|=0$. Therefore, $\left\{\mathcal{S}_{n}\right\}$ is the Cauchy sequence. Hence, it shows the above-mentioned result. 
Theorem 6 The series solution for Eq. (10) is defined in (26), then the maximum absolute error is

$$
\left\|u(x, y, t)-\sum_{n=0}^{M} u_{n}(x, y, t)\right\| \leq \frac{\lambda_{1}^{M+1}}{1-\lambda_{1}}\left\|u_{0}(x, y, t)\right\| .
$$

Proof With the assistance of Eq. (52) we have

$$
\left\|u(x, y, t)-\mathcal{S}_{n}\right\|=\lambda^{m+1}\left(\frac{1-\lambda^{n-m}}{1-\lambda}\right)\left\|u_{0}(x, y, t)\right\| .
$$

But $0<\lambda<0 \Rightarrow 1-\lambda^{n-m}<1$. Hence, we have

$$
\left\|u(x, y, t)-\sum_{n=0}^{M} u_{n}(x, t)\right\| \leq \frac{\lambda^{M+1}}{1-\lambda}\left\|u_{0}(x, y, t)\right\| .
$$

This proves the required result.

\section{Numerical results and discussion}

In this part, we consider two different cases of the FEFK equation to find the approximated analytical solution using $q$-HATM, in which the equation is associated with Mittag-Leffler kernel.

Case 1: Consider a homogeneous FEFK equation of the form

$$
{ }_{a}^{A B C} D_{t}^{\alpha} u(x, y, t)+\mu \Delta^{2} u-\Delta u+u^{3}-u=0, \quad 0<\alpha \leq 1,
$$

with the initial condition

$$
u(x, y, 0)=\sin (x) \sin (y)
$$

Case 2: Consider a non-homogeneous FEFK equation of the form

$$
{ }_{a}^{A B C} D_{t}^{\alpha} u(x, y, t)+\mu \Delta^{2} u-\Delta u+u^{3}-u=g(x, y, t), \quad 0<\alpha \leq 1,
$$

with the initial condition defined in Eq. (54). Here the non-homogeneous term is given by $g(x, y, t)=4 \mu \sin (x) \sin (y) e^{-t}+\left(\sin (x) \sin (y) e^{-t}\right)^{3}$. The corresponding exact solution for Eq. (55) is presented as follows:

$$
u(x, y, t)=\sin (x) \sin (y) e^{-t}
$$

Here, we demonstrate the exactness of the future scheme with distinct fractional order. In Fig. 1, we capture the nature of achieved solution for the homogeneous case of FEFK equation defined in Case 1 with distinct fractional order (i.e. $\alpha=0.5,0.75$ and 1 ) in terms of 2D and 3D plots. Similarly, for Case 2 the surfaces of $q$-HATM solution, analytical solution and absolute error are shown in Fig. 3. The variation of attained solution for various fractional order is presented in Fig. 4 for FEFK equation defined in the second case. As related to homotopy parameter $(\hbar)$ and at different fractional order for both cases, we present 


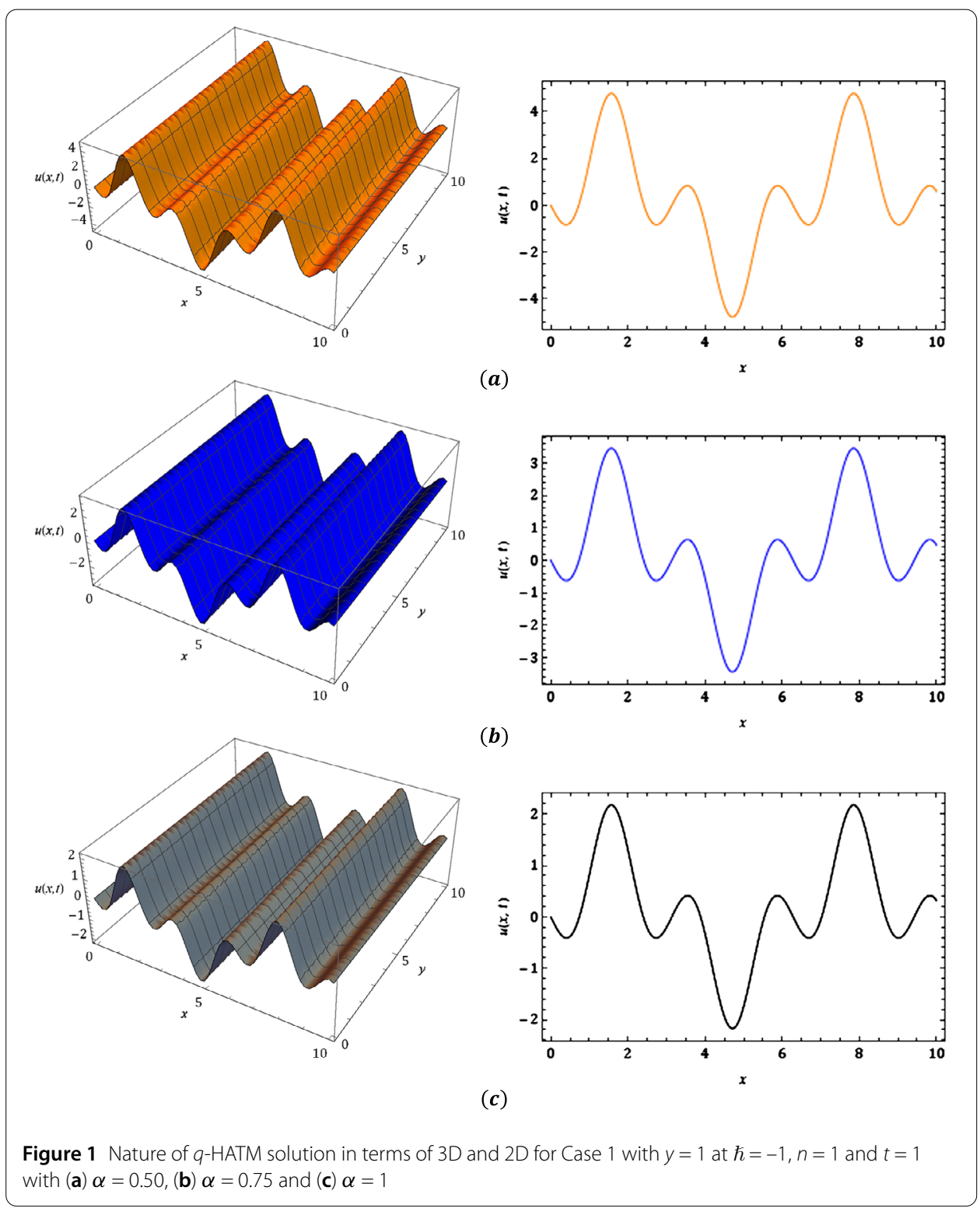

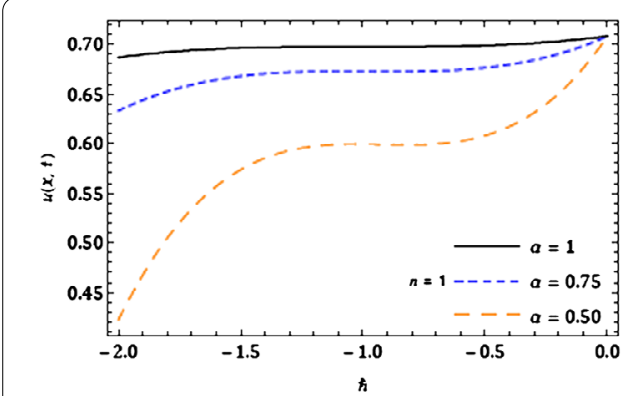

(a)

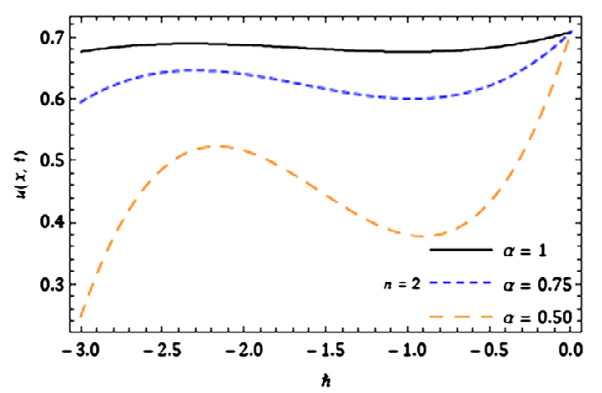

(b)

Figure $2 \hbar$-curves for $u(x, t)$ defined in Case 1 with distinct $\alpha$ at $x=1, y=1, n=1$ and $t=0.01$ with $(\mathbf{a}) n=1$ and (b) $n=2$ 


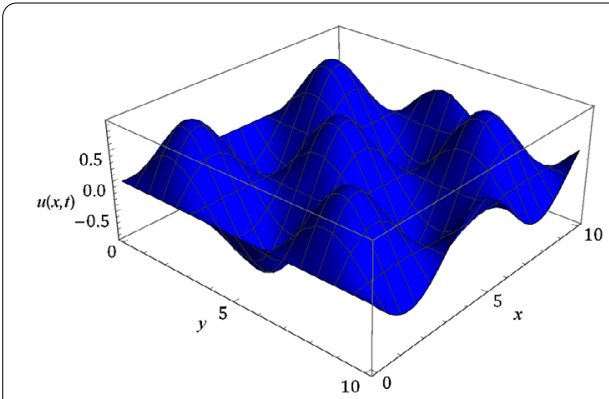

(a)

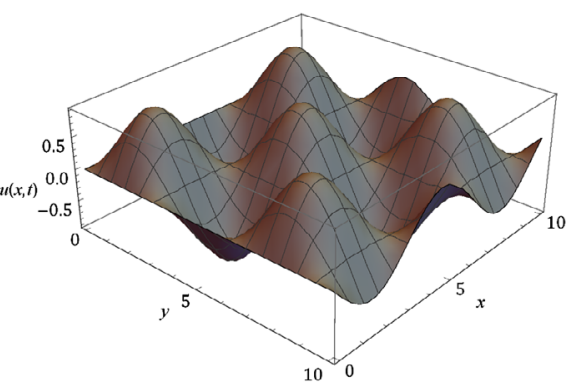

(b)

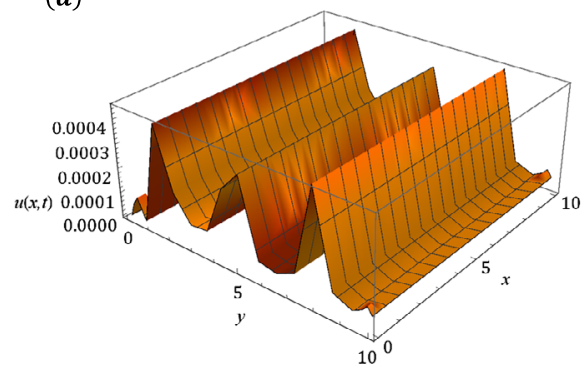

(c)

Figure 3 Surfaces (a) $q$-HATM solution (b) analytical solution (c) absolute error defined in Case 2 at $\hbar=-1$, $n=1, t=0.1$ and $\alpha=1$

Figure 4 Nature of the obtained solution defined in Case 2 with different $\alpha$ for $\hbar=-1, n=1, y=1$ and $x=1$

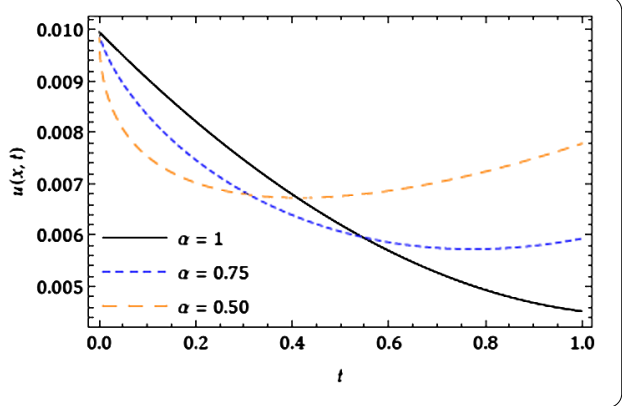

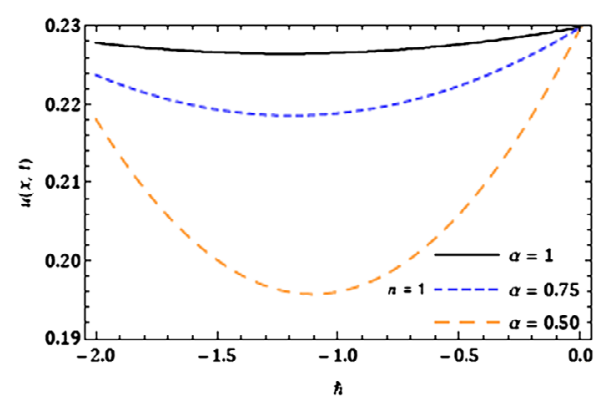

$(\boldsymbol{a})$

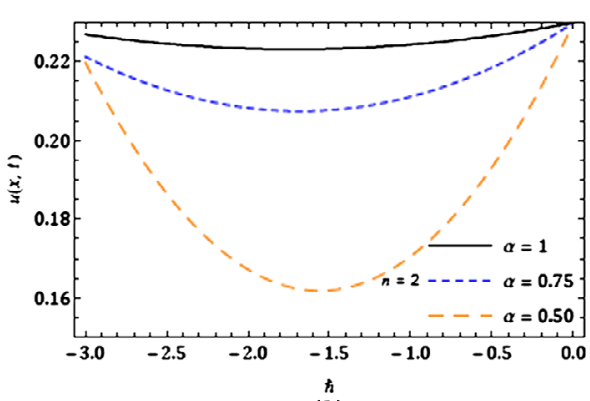

$(\boldsymbol{b})$

Figure $5 \hbar$-curves for $q$-HATM solution considered in Case 2 with different $\alpha$ for $x=1, y=1$ and $t=0.01$ with (a) $n=1$ and (b) $n=2$

the behaviour of the achieved solution respectively in Fig. 2 and Fig. 5. These curves aid to control and adjust the region of the convergence for $q$-HATM solution. Meanwhile, the horizontal line in the plots represents the convergence region. For a particular $\hbar$, the 
Table 1 Numerical illustration for the $q$-HATM solution of FEFK equation defined in Case 2 for distinct $\alpha$ at $n=1, \hbar=1$ and $y=0.1$

\begin{tabular}{|c|c|c|c|c|c|}
\hline$x$ & $t$ & $\alpha=0.7$ & $\alpha=0.8$ & $\alpha=0.9$ & $\alpha=1$ \\
\hline \multirow[t]{4}{*}{0.25} & 0.025 & $1.40637 \times 10^{-3}$ & $6.71262 \times 10^{-4}$ & $2.62076 \times 10^{-4}$ & $2.37588 \times 10^{-5}$ \\
\hline & 0.050 & $2.07408 \times 10^{-3}$ & $9.74998 \times 10^{-4}$ & $3.96960 \times 10^{-4}$ & $4.46453 \times 10^{-5}$ \\
\hline & 0.075 & $2.57439 \times 10^{-3}$ & $1.17156 \times 10^{-3}$ & $4.89850 \times 10^{-4}$ & $6.18806 \times 10^{-5}$ \\
\hline & 0.1 & $2.98624 \times 10^{-3}$ & $1.30695 \times 10^{-3}$ & $5.58417 \times 10^{-4}$ & $7.48574 \times 10^{-5}$ \\
\hline \multirow[t]{4}{*}{0.50} & 0.025 & $2.72578 \times 10^{-3}$ & $1.30527 \times 10^{-3}$ & $5.09260 \times 10^{-4}$ & $4.57329 \times 10^{-5}$ \\
\hline & 0.050 & $4.02071 \times 10^{-3}$ & $1.92679 \times 10^{-3}$ & $7.86281 \times 10^{-4}$ & $7.89672 \times 10^{-5}$ \\
\hline & 0.075 & $4.99163 \times 10^{-3}$ & $2.38024 \times 10^{-3}$ & $1.00472 \times 10^{-3}$ & $9.24355 \times 10^{-5}$ \\
\hline & 0.1 & $5.79138 \times 10^{-3}$ & $2.76164 \times 10^{-3}$ & $1.20515 \times 10^{-3}$ & $8.00485 \times 10^{-5}$ \\
\hline \multirow[t]{4}{*}{0.75} & 0.025 & $3.87642 \times 10^{-3}$ & $1.86469 \times 10^{-3}$ & $7.26833 \times 10^{-4}$ & $6.44136 \times 10^{-5}$ \\
\hline & 0.050 & $5.71957 \times 10^{-3}$ & $2.81353 \times 10^{-3}$ & $1.15165 \times 10^{-3}$ & $9.73346 \times 10^{-5}$ \\
\hline & 0.075 & $7.10277 \times 10^{-3}$ & $3.60183 \times 10^{-3}$ & $1.53830 \times 10^{-3}$ & $7.70330 \times 10^{-5}$ \\
\hline & 0.1 & $8.24315 \times 10^{-3}$ & $4.37972 \times 10^{-3}$ & $1.95701 \times 10^{-3}$ & $1.48722 \times 10^{-5}$ \\
\hline \multirow[t]{4}{*}{1} & 0.025 & $4.78660 \times 10^{-3}$ & $2.31329 \times 10^{-3}$ & $9.00816 \times 10^{-4}$ & $7.87386 \times 10^{-5}$ \\
\hline & 0.050 & $7.06455 \times 10^{-3}$ & $3.56805 \times 10^{-3}$ & $1.46487 \times 10^{-3}$ & $1.01035 \times 10^{-4}$ \\
\hline & 0.075 & $8.77561 \times 10^{-3}$ & $4.72501 \times 10^{-3}$ & $2.03957 \times 10^{-3}$ & $2.54775 \times 10^{-5}$ \\
\hline & 0.1 & $1.01876 \times 10^{-2}$ & $5.98686 \times 10^{-3}$ & $2.72760 \times 10^{-3}$ & $1.83073 \times 10^{-4}$ \\
\hline
\end{tabular}

obtained solution swiftly inclines towards analytical solution. Further, the numerical simulation has been illustrated for the non-homogeneous case proposed problem in distinct fractional order. The presented numerical study elucidates that as the order tends to the classical case, the obtained solution gets near to the analytical solution, also it confirms the exactness of the applied computational scheme.

\section{Conclusion}

In this study, we found the solution for FEKZ equation and presented the corresponding consequence by using the $q$-HATM. The present investigation confirms its competence while examining the real word problems; this is due to the considered fractional-order $\mathrm{AB}$ derivatives being defined with the help of Mittag-Leffler function. This function is nonsingular and non-local kernel in nature. For the achieved solution, we considered fixed point hypothesis to illustrate the existence and uniqueness. Further, the novelty of the considered scheme is that it did not necessitate any perturbation, discretization or conversion while finding the solution for the nonlinear problems. The present analysis shows that FEKZ equation conspicuously depends on the time instance and history. These essential properties are effectively and systematically illustrated with the help of generalised calculus. More precisely, the considered nonlinear model can be effectively and accurately analysed and exemplified with the help of newly introduced and nurtured novel numerical methods illustrated in $[65,66]$, and we consider these methods for the future work to analyse the numerous class of nonlinear models. Finally, we can accomplish that the projected technique is more effective and extremely methodical while exemplifying the diverse and interesting class of complex phenomena defined with nonlinear problems existing in science and technology.

Acknowledgements

Not applicable.

Funding

No specific funding received for this work. 
Ethics approval and consent to participate

Not applicable.

\section{Competing interests}

The authors declare that they have no competing interests.

\section{Consent for publication}

Not applicable.

\section{Authors' contributions}

The main idea of this paper was proposed by GGP and PV. JS, DK and IK prepared the manuscript initially and performed all the steps of the proofs in this research. All authors read and approved the final manuscript.

\section{Author details}

'Department of Mathematics, Karnatak University, Dharwad, India. ${ }^{2}$ Department of Mathematics, Faculty of Science, Davangere University, Davangere, India. ${ }^{3}$ Department of Mathematics, JECRC University, Jaipur, India. ${ }^{4}$ Faculty of Mathematics and Statistics, Ton DucThang University, Ho Chi Minh City, Vietnam. ${ }^{5}$ Department of Mathematics, University of Rajasthan, Jaipur, India.

\section{Publisher's Note}

Springer Nature remains neutral with regard to jurisdictional claims in published maps and institutional affiliations.

\section{Received: 13 January 2020 Accepted: 3 April 2020 Published online: 23 April 2020}

\section{References}

1. Liouville, J: Memoire sur quelques questions de geometrie et de mecanique, et sur un nouveau genre de calcul pour resoudreces questions. J. Éc. Polytech. 13, 1-69 (1832)

2. Riemann, G.F.B.: Versuch Einer Allgemeinen Auffassung der Integration und Differentiation, Gesammelte Mathematische Werke. Teubner, Leipzig (1896)

3. Caputo, M.: Elasticita e Dissipazione. Zanichelli, Bologna (1969)

4. Miller, K.S., Ross, B.: An Introduction to Fractional Calculus and Fractional Differential Equations. Wiley, New York (1993)

5. Podlubny, I.: Fractional Differential Equations. Academic Press, New York (1999)

6. Kilbas, A.A., Srivastava, H.M., Trujillo, J.J.: Theory and Applications of Fractional Differential Equations. Elsevier, Amsterdam (2006)

7. Baleanu, D., Wu, G.C., Zeng, S.D.: Chaos analysis and asymptotic stability of generalized Caputo fractional differential equations. Chaos Solitons Fractals 102, 99-105 (2017)

8. Veeresha, P., Prakasha, D.G., Baskonus, H.M.: Solving smoking epidemic model of fractional order using a modified homotopy analysis transform method. Math. Sci. 13(2), 115-128 (2019)

9. Esen, A., Sulaiman, T.A., Bulut, H., Baskonus, H.M.: Optical solitons and other solutions to the conformable space-time fractional Fokas-Lenells equation. Optik 167, 150-156 (2018)

10. Baleanu, D., Guvenc, Z.B., Machado, J.A.T.: New Trends in Nanotechnology and Fractional Calculus Applications Springer, Dordrecht (2010)

11. Veeresha, P., Prakasha, D.G., Baskonus, H.M.: New numerical surfaces to the mathematical model of cancer chemotherapy effect in Caputo fractional derivatives. Chaos 29. Article ID 013119 (2019). https://doi.org/10.1063/1.5074099

12. Gao, W., Veeresha, P., Prakasha, D.G., Baskonus, H.M., Yel, G.: A powerful approach for fractional Drinfeld-Sokolov-Wilson equation with Mittag-Leffler law. Alex. Eng. J. 58, 1301-1311 (2019)

13. Veeresha, P., Prakasha, D.G., Baskonus, H.M.: An efficient technique for a fractional-order system of equations describing the unsteady flow of a polytropic gas. Pramana J. Phys. 93, Article ID 75 (2019). https://doi.org/10.1007/s12043-019-1829-9

14. Veeresha, P., Prakasha, D.G., Baleanu, D.: An efficient numerical technique for the nonlinear fractional Kolmogorov-Petrovskii-Piskunov equation. Mathematics 7(3), Article ID 265 (2019). https://doi.org/10.3390/math7030265

15. Ziane, D., Cherif, M.H., Baleanu, D., Belghaba, K.: Exact solution for nonlinear local fractional partial differential equation. J. Appl. Comput. Mech. 6(2), 200-208 (2020)

16. Kumar, D., Singh, J., Baleanu, D.: On the analysis of vibration equation involving a fractional derivative with Mittag-Leffler law. Math. Methods Appl. Sci. 43(1), 443-457 (2020)

17. Veeresha, P., Prakasha, D.G.: An efficient technique for two-dimensional fractional order biological population model. Int. J. Model. Simul. Sci. Comput. (2020). https://doi.org/10.1142/S1793962320500051

18. Baleanu, D., Jajarmi, A., Sajjadi, S.S., Mozyrska, D.: A new fractional model and optimal control of a tumor-immune surveillance with non-singular derivative operator. Chaos 29(8), Article ID 083127 (2019). https://doi.org/10.1063/1.5096159

19. Jajarmi, A., Baleanu, D., Sajjadi, S.S., Asad, J.H.: A new feature of the fractional Euler-Lagrange equations for a coupled oscillator using a nonsingular operator approach. Front. Phys. 7, Article ID 196 (2019). https://doi.org/10.3389/fphy.2019.00196

20. Veeresha, P., Prakasha, D.G., Qurashi, M.A., Baleanu, D.: A reliable technique for fractional modified Boussinesq and approximate long wave equations. Adv. Differ. Equ. 2019, Article ID 253 (2019). https://doi.org/10.1186/s13662-019-2185-2

21. Yıldiz, T.A., Jajarmi, A., Y.ldız, B., Baleanu, D.: New aspects of time fractional optimal control problems within operators with nonsingular kernel. Discrete Contin. Dyn. Syst., Ser. S 13(3), 407-428 (2020) 
22. Veeresha, P., Prakasha, D.G.: A novel technique for $(2+1)$-dimensional time-fractional coupled Burgers equations. Math. Comput. Simul. 166, 324-345 (2019)

23. Jajarmi, A., Arshad, S., Baleanu, D.: A new fractional modelling and control strategy for the outbreak of dengue fever. Physica A 535, Article ID 122524 (2019). https://doi.org/10.1016/j.physa.2019.122524

24. Jajarmi, A., Ghanbari, B., Baleanu, D.: A new and efficient numerical method for the fractional modelling and optimal control of diabetes and tuberculosis co-existence. Chaos 29(9), Article ID 093111 (2019). https://doi.org/10.1063/1.5112177

25. Baleanu, D., Asad, J.H., Jajarmi, A.: New aspects of the motion of a particle in a circular cavity. Proc. Rom. Acad., Ser. A: Math. Phys. Tech. Sci. Inf. Sci. 19(2), 361-367 (2018)

26. Mohammadi, F., Moradi, L., Baleanu, D., Jajarmi, A.: A hybrid functions numerical scheme for fractional optimal control problems: application to non-analytic dynamical systems. J. Vib. Control 24(21), 5030-5043 (2018)

27. Baleanu, D., Asad, J.H., Jajarmi, A.: The fractional model of spring pendulum: new features within different kernels Proc. Rom. Acad., Ser. A: Math. Phys. Tech. Sci. Inf. Sci. 19(3), 447-454 (2018)

28. Veeresha, P., Prakasha, D.G., Kumar, D.: An efficient technique for nonlinear time-fractional Klein-Fock-Gordon equation. Appl. Math. Comput. 364, Article ID 124637 (2020). https://doi.org/10.1016/j.amc.2019.124637

29. Bhatter, S., Mathur, A., Kumar, D., Singh, J.: A new analysis of fractional Drinfeld-Sokolov-Wilson model with exponential memory. Physica A 537, Article ID 122578 (2020)

30. Prakasha, D.G., Veeresha, P., Rawashdeh, M.S.: Numerical solution for (2+1)-dimensional time-fractional coupled burger equations using fractional natural decomposition method. Math. Methods Appl. Sci. 42(10), 3409-3427 (2019)

31. Coullet, P., Elphick, C., Repaux, D.: Nature of spatial chaos. Phys. Rev. Lett. 58, 431-434 (1987)

32. Dee, G.T., Saarloos, W.V.: Bistable systems with propagating fronts leading to pattern formation. Phys. Rev. Lett. 60, 2641-2644 (1988)

33. Saarloos, W.V.: Front propagation into unstable states. II. Linear versus nonlinear marginal stability and rate of convergence. Phys. Rev. A 39, 6367-6389 (1989)

34. Danumjaya, P., Pani, A.K.: Orthogonal cubic spline collocation method for the extended Fisher-Kolmogorov equation J. Comput. Appl. Math. 174, 101-117 (2005)

35. Liu, F., Zhao, X., Liu, B.: Fourier pseudo-spectral method for the extended Fisher-Kolmogorov equation in two dimensions. Adv. Differ. Equ. 2017, Article ID 94 (2017). https://doi.org/10.1186/s13662-017-1154-x

36. Hornreich, R.M., Luban, M., Shtrikman, S.: Critical behaviour at the onset of k-space instability at the line. Phys. Rev. Lett. 35, 1678-1681 (1975)

37. Aronson, D.G., Weinberger, H.F.: Multidimensional nonlinear diffusion arising in population genetics. Adv. Math. 30, $33-67(1978)$

38. Zhu, G.: Experiments on director waves in nematic liquid crystals. Phys. Rev. Lett. 49, 1332-1335 (1982)

39. Caputo, M., Fabrizio, M.: A new definition of fractional derivative without singular kernel. Prog. Fract. Differ. Appl. 1(2), 73-85 (2015)

40. Atangana, A., Baleanu, D.: New fractional derivatives with non-local and non-singular kernel theory and application to heat transfer model. Therm. Sci. 20, 763-769 (2016)

41. Liao, S.J.: Homotopy analysis method and its applications in mathematics. J. Basic Sci. Eng. 5(2), 111-125 (1997)

42. Liao, S.J.: Homotopy analysis method: a new analytic method for nonlinear problems. Appl. Math. Mech. 19, 957-962 (1998)

43. Singh, J., Kumar, D., Swroop, R.: Numerical solution of time- and space-fractional coupled Burgers' equations via homotopy algorithm. Alex. Eng. J. 55(2), 1753-1763 (2016)

44. Srivastava, H.M., Kumar, D., Singh, J.: An efficient analytical technique for fractional model of vibration equation. Appl. Math. Model. 45, 192-204 (2017)

45. Prakasha, D.G., Veeresha, P., Baskonus, H.M.: Two novel computational techniques for fractional Gardner and Cahn-Hilliard equations. Comput. Math. Methods 1(2), Article ID e1021 (2019). https://doi.org/10.1002/cmm4.1021

46. Bulut, H., Kumar, D., Singh, J., Swroop, R., Baskonus, H.M.: Analytic study for a fractional model of HIV infection of CD4+ T lymphocyte cells. Math. Nat. Sci. 2(1), 33-43 (2018)

47. Veeresha, P., Prakasha, D.G.: Solution for fractional Zakharov-Kuznetsov equations by using two reliable techniques. Chin. J. Phys. 60, 313-330 (2019)

48. Kumar, D., Singh, J., Baleanu, D.: A new analysis for fractional model of regularized long-wave equation arising in ion acoustic plasma waves. Math. Methods Appl. Sci. 40, 5642-5653 (2017)

49. Singh, J., Kumar, D., Swroop, R., Kumar, S.: An efficient computational approach for time-fractional Rosenau-Hyman equation. Neural Comput. Appl. 30(10), 3063-3070 (2018)

50. Veeresha, P., Prakasha, D.G., Baskonus, H.M.: Novel simulations to the time-fractional Fisher's equation. Math. Sci. 13(1), 33-42 (2019)

51. Prakash, A., Veeresha, P., Prakasha, D.G., Goyal, M.: A homotopy technique for fractional order multi-dimensional telegraph equation via Laplace transform. Eur. Phys. J. Plus 134, Article ID 19 (2019). https://doi.org/10.1140/epjp/i2019-12411-y

52. Yeun, Y.L.: Heteroclinic solutions for the extended Fisher-Kolmogorov equation. J. Math. Anal. Appl. 407, 119-129 (2013)

53. Kadri, T., Omrani, K.: A second-order accurate difference scheme for an extended Fisher-Kolmogorov equation. Comput. Math. Appl. 61, 451-459 (2011)

54. You, H., Yuan, R., Zhang, Z.: Attractor bifurcation for extended Fisher-Kolmogorov equation. Abstr. Appl. Anal. 2013, Article ID 365436 (2013). https://doi.org/10.1155/2013/365436

55. Llibre, J., Messias, M., Silva, P.R.D.: Global dynamics of stationary solutions of the extended Fisher-Kolmogorov equation. J. Math. Phys. 52, Article ID 112701 (2011). https://doi.org/10.1063/1.3657425

56. Peletier, L.A., Troy, W.C.: Spatial patterns described by the extended Fisher-Kolmogorov equation: periodic solutions. SIAM J. Math. Anal. 28(6), 1317-1353 (1997)

57. Singh, J., Kumar, D., Hammouch, Z., Atangana, A.: A fractional epidemiological model for computer viruses pertaining to a new fractional derivative. Appl. Math. Comput. 316, 504-515 (2018) 
58. Veeresha, P., Prakasha, D.G.: Solution for fractional generalized Zakharov equations with Mittag-Leffler function. Results Eng. 5, Article ID 100085 (2020). https://doi.org/10.1016/j.rineng.2019.100085

59. Atangana, A., Alkahtani, B.T: Analysis of the Keller-Segel model with a fractional derivative without singular kernel. Entropy 17, 4439-4453 (2015)

60. Atangana, A., Alkahtani, B.T.: Analysis of non-homogenous heat model with new trend of derivative with fractional order. Chaos Solitons Fractals 89, 566-571 (2016)

61. Prakasha, D.G., Veeresha, P., Singh, J.: Fractional approach for equation describing the water transport in unsaturated porous media with Mittag-Leffler kernel. Front. Phys. 7, Article ID 193 (2019). https://doi.org/10.3389/fphy.2019.00193

62. Veeresha, P., Prakasha, D.G., Baskonus, H.M.: An efficient technique for coupled fractional Whitham-Broer-Kaup equations describing the propagation of shallow water waves. In: International Conference on Computational Mathematics and Engineering Sciences. Advances in Intelligent Systems and Computing, vol. 1111, pp. 49-75. Springer, Cham (2020). https://doi.org/10.1007/978-3-030-39112-6_4

63. Kumar, D., Agarwal, R.P., Singh, J.: A modified numerical scheme and convergence analysis for fractional model of Lienard's equation. J. Comput. Appl. Math. 339, 405-413 (2018)

64. Veeresha, P., Prakasha, D.G., Baleanu, D.: Analysis of fractional Swift-Hohenberg equation using a novel computational technique. Math. Methods Appl. Sci. 43(4), 1970-1987 (2020). https://doi.org/10.1002/mma.6022

65. Hajipour, M., Jajarmi, A., Baleanu, D.: On the accurate discretization of a highly nonlinear boundary value problem. Numer. Algorithms 79(3), 679-695 (2018)

66. Hajipour, M., Jajarmi, A., Malek, A., Baleanu, D.: Positivity-preserving sixth-order implicit finite difference weighted essentially non-oscillatory scheme for the nonlinear heat equation. Appl. Math. Comput. 325, 146-158 (2018)

\section{Submit your manuscript to a SpringerOpen ${ }^{\circ}$ journal and benefit from:}

- Convenient online submission

- Rigorous peer review

- Open access: articles freely available online

- High visibility within the field

Retaining the copyright to your article

Submit your next manuscript at $\boldsymbol{~ s p r i n g e r o p e n . c o m ~}$ 